significantly influence the outcome. Hypertension was present in 18 patients; 10 patients had type 2 diabetes (T2DM), 10 obstructive sleep apnoea (OSA), seven asthma, five hypothyroidism and two chronic obstructive pulmonary disease. The absence of OSA was associated with successful target weight loss. This did not reach statistical significance (24 patients without OSA achieved their target excess body weight loss; $\mathrm{p}=0.079$ ).

Conclusion The majority of patients in the present study achieved the target weight loss quoted in the literature. The presence of OSA appears to negatively influence the achievement of pre-operative excess body weight loss.

Competing interests None declared.

\section{PMO-050 A SURVEY OF GP'S KNOWLEDGE AND ATTITUDES TOWARDS BARIATRIC SURGERY IN SCOTLAND}

doi:10.1136/gutjnl-2012-302514b.50

\author{
1J Kynaston, ${ }^{*}{ }^{1} \mathrm{~A}$ Mitchell, ${ }^{2} \mathrm{D}$ Bruce. ${ }^{1}$ Aberdeen Royal infirmary, Aberdeen, UK; \\ ${ }^{2}$ Aberdeen Surgical, Aberdeen, UK
}

Introduction $25 \%$ of Scotland's population are obese. The benefits of bariatric surgery are established however General Practitioners (GP's) influence equality of access to this treatment. This study examines GP's knowledge and attitudes to bariatric surgery in Scotland.

Methods An electronic questionnaire-based study was emailed to all 902 GP's within the NHS Highland, Grampian and Tayside health board regions in Scotland. The questionnaire was piloted with a convenience sample of GP's. Respondents were asked their age, sex and eight questions examining their knowledge, experience and opinions regarding bariatric surgery.

Results 230 GP's completed the survey (25.4\% response), a further 11 incomplete submissions were not analysed. 60\% $(n=139)$ of respondents were female. $93 \%$ of GPs acknowledge they often encounter weight management issues. $62 \% \quad(n=142)$ of GPs acknowledge bariatric surgery has an important role in weight management and 69\% ( $n=159)$ acknowledge it's importance in the management of the obesity related co-morbidities. 30\% $(n=68)$ of GPs are not aware of NICE/SIGN guidelines and $57 \%(n=132)$ are not aware of their local referral criteria. $40 \%(n=103)$ of GPs have never referred. Of those that have, $34 \%(n=43)$ of referrals were to the private sector. $76 \%(n=174)$ of GPs are not comfortable managing patients who have undergone bariatric surgery. $8 \%$ $(n=17)$ of GPs believe the primary role of bariatric surgery is cosmetic. Of these, $47 \%(n=9)$ are aged $25-40$ years.

Conclusion Weight management issues are common in primary care and most GP's support bariatric surgery. However a third of GP's are unaware of national guidelines and half do not know how to refer to their local service in Northern Scotland. Most GP's are not comfortable providing long-term care after bariatric surgery. These findings suggest GP's require education and support to allow their patients access to bariatric surgery.

Competing interests None declared.

\section{PMO-051 PRE-OPERATIVE DIETARY WEIGHT LOSS DOES NOT CORRELATE WITH BETTER POST-OPERATIVE OUTCOMES FROM LAPAROSCOPIC ADJUSTABLE GASTRIC BANDING}

doi:10.1136/gutjnl-2012-302514b.51

M Singh, J Plowright, ${ }^{*}$ P Super. Department of General Surgery, NHS, Birmingham, UK

Introduction Patients considered for laparoscopic adjustable gastric banding (LAGB) are encouraged to lose weight pre-operatively, based on the hypothesis that good pre-operative dietary weight loss correlates with a favourable post-operative outcome and allows safe surgery. This study aims to assess whether pre-operative weight loss is a true predictor of post-operative weight loss in patients who underwent LAGB for morbid obesity.

Methods A retrospective analysis of patients who underwent LAGB in 2007 at our institution by a single surgeon, using actual body weight loss pre-operatively (from patient's BMI at the bariatric surgical clinic vs their BMI on the pre-operative day) and then comparing this to their BMI at 1 and 2 years post-operatively.

Results 69 patients were included in the study $(M=23, F=46, M$ : $\mathrm{F}=1: 2$ ), with a mean age of 45.7 years (Range 19-64). The average $\mathrm{BMI}$ at the bariatric surgical clinic was 54.01 (Range $=38-72$ ). The average BMI on the pre-operative day was 52.13 (Range 33-70), which reflected in a mean reduction in BMI of 1.88 (or $4.86 \mathrm{~kg}$ ) (Range -5-19) or a mean excess percent BMI loss (EBL) of $7.4 \mathrm{~kg}$ / $\mathrm{m}^{2}$. An analysis of their 1- and 2-year post-operative weight loss compared to their weights from clinic was then performed. At 1 year post-operatively, the mean reduction in BMI was 11.1 (Range $=-5-27)$ or an EBL of $33.6 \mathrm{~kg} / \mathrm{m}^{2}$. At 2 years post-operatively, the mean reduction in BMI was 13.29 (Range $=-1-35$ ) or an EBL of $41.5 \mathrm{~kg} / \mathrm{m}^{2}$. Correlation between pre-operative weight loss vs weight lost at 1 and 2 years post LAGB was performed using the Spearman Rank Correlation, as the data were not normally distributed. At 1 year post-operatively, the Spearman Rank Correlation was 0.154 (95\% CI -0.094 to 0.383 ) with a $p$ value of 0.208 (no statistical significant correlation). At 2 years post-operatively, the Spearman Rank Correlation was 0.069 (95\% CI -0.177 to 0.307 ) with a $p$ value of 0.573 (no statistical significant correlation). Scatter plot graphs of individual pre-operative weight loss data vs year 1 and year 2 weight loss data confirmed there was no linear correlation.

Conclusion Pre-operative dietary weight loss does not correlate with better outcomes following laparoscopic adjustable gastric banding.

Competing interests None declared.

\section{PM0-052 SLEEVE GASTRECTOMY FOR OBESITY: A SYSTEMATIC REVIEW}

doi:10.1136/gutjnl-2012-302514b.52

J Al Shakarchi.* Russells Hall Hospital, Dudley, UK

Introduction Sleeve gastrectomy has become an accepted and popular bariatric procedure world wide. Weight loss is achieved through restrictive and endocrine mechanisms. We aimed to evaluate the efficiency of excess weight loss by sleeve gastrectomy and to compare it to other bariatric procedures.

Methods We searched the Cochrane Database of Systematic Reviews and Pubmed for studies assessing the efficiency of sleeve gastrectomy. Strict inclusion criteria and thorough appraisal of the reviews was required to ensure comparability of the included papers. Results Our dataset comprised of individual data on 1845 patients from 27 different studies. The median difference in excess body weight loss across the 27 studies which reported weight loss was a significant difference of $56.2 \%$ (range 33-85). The mean mortality rate reported from the studies was $0.16 \%$. The major postoperative complication rate ranged from $0 \%$ to $23.8 \%$.

Conclusion From the data we have gathered it may be reasonable to conclude that sleeve gastrectomy is an efficient procedure which achieves comparable if not better excess weight loss to both gastric bypass and banding. It may be achieved safely and has similar complication rates to other bariatric procedures. However there is currently little data assessing long term outcomes of the procedure.

Competing interests None declared. 